\title{
Avaliação da Estabilidade de um xampu Produzido com Adição de Óleo Essencial de Alecrim (Rosmarinus officinalis)
}

\author{
Evaluation of the stability of a xampu produced with the addition of essential oil of rosemary \\ (Rosmarinus officinalis) \\ Guilherme do Canto Pereira ${ }^{\dagger}$, Suellen Cristine de Moura Murat ${ }^{\ddagger}$, Bruna de Souza Magalhães ${ }^{\dagger}$, Byanca \\ Ribeiro Benevenuto§, Larissa Dionísio da Silva ${ }^{\ddagger}$, Rejane dos Santos Garcia Ribeiro", Cristiane de \\ Souza Siqueira Pereirat ${ }^{\dagger, \| *}$
}

Como citar esse artigo. Pereira,

GC; Murat, SCM; Magalhães, BS; Benevenuto, BR; da Silva, LD; Ribeiro, RSG; Pereira, CSS. Revista Teccen. 2020 Jan./Jun.; 13 (1): 02-07.

\begin{abstract}
Resumo
A indústria mundial de produtos dermocosméticos cresce a cada ano em virtude do desenvolvimento constante de novos produtos. $\mathrm{O}$ alecrim Rosmarinus officinalis possui várias propriedades e dentre estas propriedades destaca-se por manter o equilíbrio e saúde do couro cabeludo propiciando um crescimento saudável dos fios e a redução significativa da caspa. De acordo com a Agência Nacional de Vigilância Sanitária (ANVISA), para os produtos cosméticos é necessário realizar o estudo de estabilidade, para avaliar por quanto tempo o produto manterá suas qualidades físico-químicas dentro dos limites previamente estabelecidos. O teste emprega condições distintas de temperatura com o objetivo de acelerar possíveis reações entre seus componentes e o surgimento de sinais que devem ser observados e analisados conforme as características específicas do produto. O presente trabalho teve como objetivo um estudo preliminar para avaliar a estabilidade do xampu produzido com a adição do óleo essencial de alecrim, em diferentes condições de temperaturas: $5^{\circ} \mathrm{C}, 25^{\circ} \mathrm{C}$ e $40^{\circ} \mathrm{C}$. O xampu foi analisado quanto aos parâmetros físico-químicos como $\mathrm{pH}$, viscosidade e densidade e quanto ao parâmetro organoléptico de aspecto visual, por um período de 30 dias. Os resultados obtidos evidenciaram que a formulação apresentou boa tolerância em relação à estabilidade e mostrou-se estável em condições adversas. Palavras-Chave: Teste de Estabilidade, Dermocosmético, Hidrodestilação.
\end{abstract}

\begin{abstract}
The global dermocosmetic products industry grows every year due to the constant development of new products. The rosemary Rosmarinus officinalis has several properties and among these properties stands out for maintaining the balance and health of the scalp, providing a healthy growth of the hair and a significant reduction of dandruff. According to the National Health Surveillance Agency ANVISA, for cosmetic products it is necessary to carry out a stability study, which consists of carrying out the test to verify for how long the product will maintain its physico-chemical properties within the established limits. It uses extreme temperature conditions in order to accelerate possible reactions between its components and the appearance of signs that must be observed and analyzed according to the specific characteristics of the product. The present work aimed at a preliminary study to evaluate the stability of the xampuproduced with the addition of rosemary essential oil, under different temperature conditions: $5{ }^{\circ} \mathrm{C}, 25^{\circ} \mathrm{C}$ and $40{ }^{\circ} \mathrm{C}$. The xampu was analyzed for physical-chemical parameters such as $\mathrm{pH}$, viscosity and density and for the organoleptic parameter of visual aspect, for a period of 30 days. The results obtained showed that the formulation showed good tolerance in relation to stability and was stable in adverse conditions.

Keywords: Stability Test, Dermocosmetic, Hydrodistillation.
\end{abstract}

\section{Introdução}

Diversas plantas são utilizadas na elaboração de dermocosméticos, pois são conhecidas pelas suas substâncias ativas, devido as suas características antibacterianas, principalmente nos óleos essenciais, compostos por terpenos que conferem aroma e sabor característico, tendo um papel fundamental nas funções essenciais da planta (RIBEIRO, 2012).

Os óleos essenciais são extraídos de vegetais através, principalmente, da técnica de arraste a vapor e também pela prensagem do pericarpo de frutos cítricos, que no Brasil comandam o mercado de exportação. São constituídos, sobretudo de mono e sesquiterpenos e de fenilpropanoides, metabólitos que conferem suas características organolépticas. Flores, folhas, cascas, rizomas e frutos são matérias-primas para sua produção, a exemplo dos óleos essenciais de rosas, eucalipto e alecrim, canela, gengibre e laranja, respectivamente. Possuem extensa aplicação na perfumaria, cosmética, alimentos e como auxiliares em medicamentos. São

\footnotetext{
Afiliação dos autores:

$†$ Curso de Engenharia Química, Universidade de Vassouras, Vassouras - RJ, Brasil.

\$ Engenheira Química, Universidade de Vassouras - RJ, Brasil.

§ Curso de Farmácia, Universidade Federal Rural do Rio de Janeiro - Seropédica - RJ, Brasil

|| Mestrado Profissional em Ciências Ambientais, Universidade de Vassouras - RJ, Brasil.

* Email para correspondência: cristiane.pereira@universidadedevassouras.edu.br
} 
empregados principalmente como aromas, fragrâncias, fixadores de fragrâncias, em composições farmacêuticas e orais e comercializados na sua forma bruta ou beneficiada, fornecendo substâncias purificadas como o limoneno, citral, citronelal, eugenol, mentol e safrol (BIZZO, 2009).

O alecrim (Rosmarinus officinalis) é uma planta amplamente conhecida, nativa do mediterrâneo, pertencente à família botânica das Lamiaceae. Desde os tempos antigos a erva é convertida para suas propriedades medicinais. $\mathrm{O}$ alecrim é tradicionalmente usado para ajudar a aliviar a dor muscular, melhorar a memória, aumentar o sistema imunológico e circulatório, e melhora capilar (CARVALHO JUNIOR, 2004).

É mundialmente utilizado em diferentes ramos, tais como culinário, medicinal, aromaterápico e em diversas indicações farmacêuticas. Apresenta ação antibacteriana, anti-inflamatória, antisséptica, carminativa, vasodilatadora, cicatrizante e estimulante do couro cabeludo (PENTEADO E CECY, 2005).

Boix et al. (2010) identificaram como principais componentes do alecrim o $\beta$-pineno $(7,0 \%)$, mirceno (9,52\%), 1,8 cineol $(14,02 \%)$, cânfora $(33,17 \%)$ e verbenona $(8,6 \%)$ e Gachkar et al. (2007) observaram que os compostos majoritários da especiaria foram $\alpha$-pineno $(14,9 \%), 1,8$ cineol $(7,43 \%)$ e linalol $(14,9 \%)$.

$\mathrm{O}$ estudo dos vegetais e a aplicação dos seus constituintes, utilizados na forma de extratos ou na forma de substâncias ativas isoladas, tem ganhado cada vez mais espaço na indústria cosmética moderna e atual (RUIVO, 2012). O mercado brasileiro de cosméticos vem passando por grandes transformações no mundo, e consequentemente no Brasil, motivadas pela introdução de novos processos produtivos, aplicação de novas matérias primas, novas concepções de produtos, entre outras. Os xampus e os condicionadores estão entre os produtos de higiene pessoal mais procurados pelos consumidores, que estão cada vez mais exigentes sobre a composição química do produto que estão consumindo, envolvendo preocupações com seu bemestar e a qualidade do meio ambiente (DE CASTRO, 2019).

O xampu é um produto fundamental para a higienização do couro cabeludo e cabelo por sua função de remover a sujeira e o excesso de oleosidade (SCACHETI, et al., 2011). No desenvolvimento de xampus, alguns fatores devem ser levados em conta, como a baixa irritabilidade, garantindo segurança aos consumidores com sensibilidade ocular e dérmica. Alguns produtos como cremes, loções capilares e xampus são dermatológicos e são indicadas com uso tópico contra coceiras, vermelhidões, inflamações e descamações do couro cabeludo, como a caspa e a dermatite seborreica (FERREIRA, 2008).

De acordo com a estabilidade de um produto é possível avaliar seu desempenho, segurança e eficácia.
$\mathrm{O}$ estudo de estabilidade fornece indicações sobre o comportamento do produto, em determinado intervalo de tempo, frente a condições de temperatura a que possa ser submetido, desde o final da formulação até o término da validade. Produtos expostos ao consumo que apresentem problemas de estabilidade organoléptica e físico-química, além de descumprirem os requisitos técnicos de qualidade podem colocar em risco a saúde do consumidor configurando infração sanitária (BRASIL, 2004).

Os fitocosméticos, dermocosméticos e cosméticos em geral passam por classificações e inspeções de órgãos reguladores como a Agência Nacional de Vigilância Sanitária (ANVISA), exigindo rigorosos estudos de pesquisa, processos de criação e desenvolvimento, e isso inclui os testes de estabilidade, cujo principal objetivo é garanti-la durante a vida útil do produto final (ISAAC et al, 2008).

Os parâmetros que geralmente são avaliados devem ser definidos pelo formulador e dependem das características da formulação em estudo e dos componentes utilizados nesta formulação. De modo geral, avaliam-se características organolépticas (aspecto, cor, odor) e características físico-químicas (valores de $\mathrm{pH}$, viscosidade e densidade). Deve-se tomar uma amostra de referência (padrão), que em geral pode ser mantida em geladeira, estufa e temperatura ambiente, ao abrigo da luz. Em caráter complementar, podem ser também utilizadas amostras de mercado, ou outros produtos semelhantes, considerados satisfatórios no que se refere aos parâmetros avaliados (ZANON, 2010).

No presente trabalho desenvolveu-se um xampu, adicionado em sua formulação aproximadamente $2 \mathrm{~mL}$ de óleo essencial de alecrim objetivando-se realizar um estudo preliminar para avaliar a estabilidade do xampu produzido com a adição do óleo essencial de alecrim, em diferentes condições de temperaturas: $5^{\circ} \mathrm{C}, 25^{\circ} \mathrm{C} \mathrm{e} 40^{\circ} \mathrm{C}$. Utilizando os parâmetros fornecidos por uma empresa de dermocosméticos e faixas de análise de um xampu padrão do mercado. O xampu foi analisado quanto aos parâmetros físico-químicos como $\mathrm{pH}$, viscosidade e densidade e quanto ao parâmetro organoléptico de aspecto visual, por um período de 30 dias.

\section{Metodologia}

\section{- Preparo da amostra}

As amostras das folhas de alecrim foram coletadas no município de Vassouras-RJ e secas em estufas (40 $\left.{ }^{\circ} \mathrm{C}\right)$ por 3 dias. Após secagem, as folhas foram trituradas para posteriormente serem submetidas ao processo de extração do óleo essencial.

Os experimentos foram realizados no Laboratório de Engenharia Química da Universidade de Vassouras, Vassouras-RJ. 


\section{- Extração do óleo essencial}

Para a extração do óleo, utilizou-se a técnica de hidrodestilação utilizando o aparelho Clevenger. A técnica de hidrodestilação consiste na imersão do material vegetal na água sob aquecimento e fervura, formando vapores que arrastam os compostos voláteis que irão se separar da fase aquosa por decantação após a condensação (PRINS, 2006).

Cerca de $80 \mathrm{~g}$ das folhas do alecrim foram adicionadas ao Clevenger com $500 \mathrm{~mL}$ de água. A extração ocorreu por um período de 3 horas. Para o cálculo do rendimento do óleo extraído utilizou-se a seguinte Equação:

$$
\text { Rendimento do óleo (\%) }=\frac{m 2}{m 1} \times 100 \%
$$

Onde: $\mathrm{m} 2$ é a massa de óleo extraído e $\mathrm{m} 1$ a massa inicial da matéria prima.

\section{- Produção do xampu}

$\mathrm{Na}$ formulação do xampu incorporou-se cerca de $2 \mathrm{~mL}$ do óleo essencial à uma base comercial própria para produção de xampu. Além da base, a formulação consistiu dos seguintes componentes: $2,4 \mathrm{~mL}$ de essência, $1,35 \mathrm{~mL}$ de óleo vegetal e $5 \mathrm{~mL}$ de corante, para hidratação e fragrância. Após esse processo, envasou-se o produto em pequenos frascos de $20 \mathrm{~mL}$ e este foram enviados para análises de estabilidade. Para avaliação da estabilidade, as amostras foram mantidas nas temperaturas de $25{ }^{\circ} \mathrm{C}, 40{ }^{\circ} \mathrm{C}$ e $5^{\circ} \mathrm{C}$. Uma vez por semana, as amostras foram analisadas determinando-se $\mathrm{pH}$, viscosidade, densidade e avaliações visuais como cor, odor e homogeneidade, atentando-se a qualquer tipo de alteração. Este estudo ocorreu por um período de 30 dias.

\section{- Determinação da viscosidade}

Para a determinação da viscosidade, utilizou-se um viscosímetro Brookfield RV. O equipamento foi configurado utilizando uma agulha \#31 e um tempo cronometrado de 3 minutos a uma velocidade de 12 $\mathrm{RPM}$, à $25^{\circ} \mathrm{C}$. A faixa utilizada do xampu de referência é de 5.000 á $30.000 \mathrm{cPs}$.

\section{- Determinação da densidade}

Empregou-se o método do picnômetro, que demonstra a densidade relativa em relação à água, de acordo com a Farmacopeia Brasileira (1988). Após calibração do equipamento, foi realizada a medição da densidade da amostra utilizando a faixa do xampu de referência de $0,950 \mathrm{~g} / \mathrm{mL}$ a $1,100 \mathrm{~g} / \mathrm{mL}$.

\section{- Determinação do pH}

$\mathrm{O} \mathrm{pH}$ foi determinado à $25^{\circ} \mathrm{C}$, utilizando $\mathrm{pHmetro}$ digital DIGIMED e empregando o método de eletrodo. A avaliação do valor de $\mathrm{pH}$ foi aferida com soluções tampão de referência, pH 4,0 e 7,0 (Farmacopeia Brasileira, 2000). De forma a obter o $\mathrm{pH}$ característico da formulação, para isso, coletava-se $100 \mathrm{~mL}$ da amostra em um béquer e realizava-se sua medição por potenciometria, utilizando a média de $\mathrm{pH}$ entre 5,5 - 7,0 (faixa padrão de qualidade do xampu de referência).

\section{Resultados e discussão}

As condições de extração do alecrim permitiu a obtenção do óleo essencial de alecrim de forma eficiente, que posteriormente foi adicionado à formulação do xampu, sendo realizada através da hidrodestilação, que utiliza água na fase vapor $\left(100^{\circ} \mathrm{C}\right)$. A Tabela 1 apresenta o rendimento do óleo essencial de alecrim obtido após o processo de extração.

Tabela 1. Rendimento do óleo essencial de alecrim

\begin{tabular}{lcc}
\hline $\begin{array}{c}\text { Massa da matéria } \\
\text { prima (g) }\end{array}$ & Massa do óleo & $\begin{array}{c}\text { Rendimento \% } \\
(\mathbf{g} / \mathbf{g})\end{array}$ \\
\hline 80 & 1,64 & 2,05 \\
\hline
\end{tabular}

Fonte: Os autores

Com relação às propriedades de estabilidade, o xampu produzido não apresentou alterações visuais e organolépticas durante o período de 30 dias, mantendose como um líquido viscoso, perolado, brilhante, de cor amarelo claro e odor característico. Tais resultados são apresentados na tabela 2 e são satisfatórios, pois a avaliação de aspecto, cor e odor é um método eficaz na percepção de anomalias e instabilidades, como alteração de aparência, homogeneidade, odores e coloração.

A viscosidade é dada como a resistência de um fluido frente ao fluxo ou movimento mecânico realizado sobre ele (CUNHA, 2009). Os resultados de viscosidade obtidos mostraram-se ser adequados para a formulação, pois o produto dever conferir aderência ao couro cabeludo. Os resultados apresentados em relação ao teste de viscosidade mostraram-se satisfatórios para os parâmetros adotados, de acordo com a faixa de viscosidade de um xampu comercial utilizado como referência (5.000 cPs á $30.000 \mathrm{cPs})$. Os resultados para viscosidade podem ser observados através do Gráfico 1.

A importância de se determinar a densidade de um xampu é que este parâmetro indica a perda de ingredientes voláteis ou a incorporação de ar. De acordo com a Tabela 3, observa-se que os resultados das análises de densidade se encontram dentro do padrão estabelecido, utilizando a faixa de um xampu comercial 
Tabela 2. Avaliação do aspecto do xampu no período de 30 dias.

\begin{tabular}{|c|c|c|}
\hline Temperatura & Dias & Aspecto \\
\hline \multirow{5}{*}{$5^{\circ} \mathrm{C}$} & 0 & $\begin{array}{l}\text { Líquido viscoso, perolado, brilhante, de cor amarelo claro e odor ca- } \\
\text { racterístico }\end{array}$ \\
\hline & 7 & $\begin{array}{l}\text { Líquido viscoso, perolado, brilhante, de cor amarelo claro e odor ca- } \\
\text { racterístico }\end{array}$ \\
\hline & 14 & $\begin{array}{l}\text { Líquido viscoso, perolado, brilhante, de cor amarelo claro e odor ca- } \\
\text { racterístico }\end{array}$ \\
\hline & 21 & $\begin{array}{l}\text { Líquido viscoso, perolado, brilhante, de cor amarelo claro e odor ca- } \\
\text { racterístico }\end{array}$ \\
\hline & 30 & $\begin{array}{l}\text { Líquido viscoso, perolado, brilhante, de cor amarelo claro e odor ca- } \\
\text { racterístico }\end{array}$ \\
\hline \multirow{5}{*}{$40^{\circ} \mathrm{C}$} & 0 & $\begin{array}{l}\text { Líquido viscoso, perolado, brilhante, de cor amarelo claro e odor ca- } \\
\text { racterístico }\end{array}$ \\
\hline & 7 & $\begin{array}{l}\text { Líquido viscoso, perolado, brilhante, de cor amarelo claro e odor ca- } \\
\text { racterístico }\end{array}$ \\
\hline & 14 & $\begin{array}{l}\text { Líquido viscoso, perolado, brilhante, de cor amarelo claro e odor ca- } \\
\text { racterístico }\end{array}$ \\
\hline & 21 & $\begin{array}{l}\text { Líquido viscoso, perolado, brilhante, de cor amarelo claro e odor ca- } \\
\text { racterístico }\end{array}$ \\
\hline & 30 & $\begin{array}{l}\text { Líquido viscoso, perolado, brilhante, de cor amarelo claro e odor ca- } \\
\text { racterístico }\end{array}$ \\
\hline \multirow{5}{*}{$25^{\circ} \mathrm{C}$} & 0 & $\begin{array}{l}\text { Líquido viscoso, perolado, brilhante, de cor amarelo claro e odor ca- } \\
\text { racterístico }\end{array}$ \\
\hline & 7 & $\begin{array}{l}\text { Líquido viscoso, perolado, brilhante, de cor amarelo claro e odor ca- } \\
\text { racterístico }\end{array}$ \\
\hline & 14 & $\begin{array}{l}\text { Líquido viscoso, perolado, brilhante, de cor amarelo claro e odor ca- } \\
\text { racterístico }\end{array}$ \\
\hline & 21 & $\begin{array}{l}\text { Líquido viscoso, perolado, brilhante, de cor amarelo claro e odor ca- } \\
\text { racterístico }\end{array}$ \\
\hline & 30 & $\begin{array}{l}\text { Líquido viscoso, perolado, brilhante, de cor amarelo claro e odor ca- } \\
\text { racterístico }\end{array}$ \\
\hline
\end{tabular}

Fonte: Os autores

Gráfico 1. Resultados da análise de viscosidade das amostras

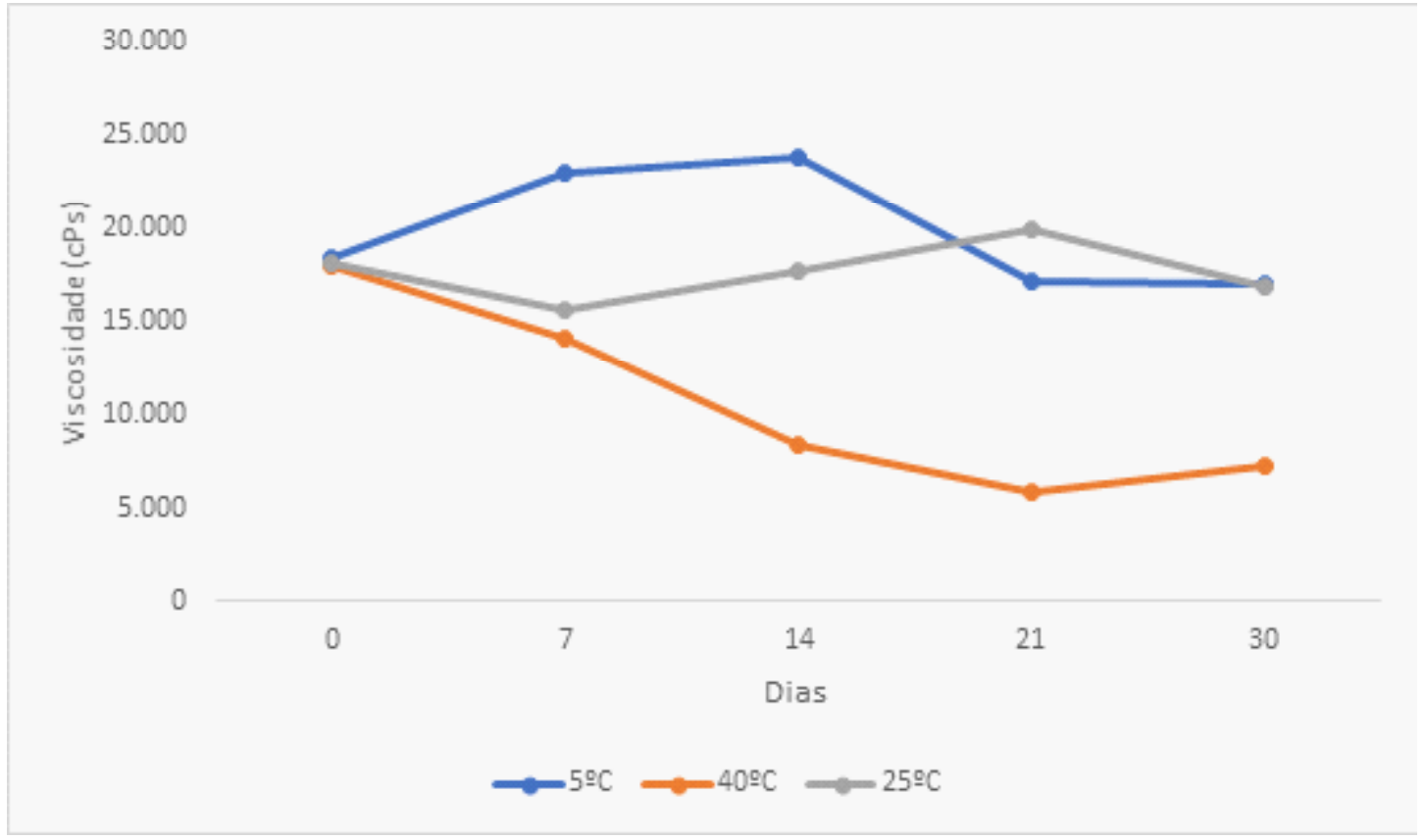

Fonte: Os autores 
Tabela 3. Avaliação da densidade no período de 30 dias

\begin{tabular}{|c|c|c|}
\hline Temperatura & Dias & $\begin{array}{c}\text { Densidade } \\
(\mathrm{g} / \mathrm{mL})\end{array}$ \\
\hline \multirow{5}{*}{$5^{\circ} \mathrm{C}$} & 0 & 1,014 \\
\hline & 7 & 1,018 \\
\hline & 14 & 1,003 \\
\hline & 21 & 1,009 \\
\hline & 30 & 1,012 \\
\hline \multirow{5}{*}{$40^{\circ} \mathrm{C}$} & 0 & 1,014 \\
\hline & 7 & 1,015 \\
\hline & 14 & 1,015 \\
\hline & 21 & 1,017 \\
\hline & 30 & 1,019 \\
\hline \multirow{5}{*}{$25^{\circ} \mathrm{C}$} & 0 & 1,014 \\
\hline & 7 & 1,014 \\
\hline & 14 & 1,012 \\
\hline & 21 & 1,010 \\
\hline & 30 & 1,008 \\
\hline
\end{tabular}

Fonte: Os autores

utilizado como referência que é de $0,950 \mathrm{~g} / \mathrm{mL}$ a 1,100 $\mathrm{g} / \mathrm{mL}$.

A determinação por potenciometria direta é de suma importância no estudo de estabilidade, devido ser um indicador de que não houve formação de compostos de degradação. Os valores de $\mathrm{pH}$ obtidos mostraram que não ocorreram alterações na formulação durante o período de avaliação, o que é o esperado não ter nenhuma tendência a subir ou abaixar muito os valores de $\mathrm{pH}$ durante o período das análises, pois alterações no $\mathrm{pH}$, indicam decomposições, presença de impurezas, assim como condições inapropriadas de armazenamento (CUNHA, 2009).

De acordo com a Tabela 4, observa-se que não ocorreram alterações significativas nos resultados do $\mathrm{pH}$.

A partir das análises físico-químicas, como $\mathrm{pH}$, densidade, aspecto e viscosidade, evidenciou-se que o xampu formulado com a adição do óleo de alecrim se manteve estável dentro dos parâmetros estabelecidos e
Tabela 4. Avaliação do pH no período de 30 dias

\begin{tabular}{|c|c|c|}
\hline Temperatura & Dias & pH \\
\hline \multirow{5}{*}{$5^{\circ} \mathrm{C}$} & 0 & 6,9 \\
\hline & 7 & 6,9 \\
\hline & 14 & 6,9 \\
\hline & 21 & 6,9 \\
\hline & 30 & 6,7 \\
\hline \multirow{5}{*}{$40^{\circ} \mathrm{C}$} & 0 & 6,9 \\
\hline & 7 & 6,9 \\
\hline & 14 & 6,9 \\
\hline & 21 & 6,8 \\
\hline & 30 & 6,8 \\
\hline \multirow{4}{*}{$25^{\circ} \mathrm{C}$} & 0 & 6,9 \\
\hline & 7 & 6,9 \\
\hline & 14 & 6,9 \\
\hline & 21 & 6,8 \\
\hline
\end{tabular}

Fonte: Os autores

comparados a um xampu, obtendo-se assim, uma boa estabilidade e um produto que possivelmente poderá apresentar ação antisséptica e auxiliar do crescimento capilar.

\section{Conclusão}

No presente estudo, realizou-se a um estudo preliminar da estabilidade de um xampu produzido com a incorporação de óleo essencial de alecrim, tendo em vista que este óleo apresenta características benéficas à saúde humana em termos de química verde.

A partir dos resultados obtidos das análises, levando-se em consideração os parâmetros de um xampu utilizado como padrão de referência presente no mercado, foi possível observar que os resultados se mostraram satisfatórios, visto que este os parâmetros analisados apresentaram estáveis nos testes de $\mathrm{pH}$, densidade, viscosidade, coloração e odor. Como perspectivas futuras, sugere-se a avaliação da composição do óleo essencial de alecrim através da técnica de cromatografia gasosa e também a realização de um estudo de 
estabilidade com análises de cromatografia líquida de alta eficiência (HPLC/CLAE) e cromatografia em fase gasosa (CG).

\section{Referências}

Brasil. (2004). Guia de estabilidade de produtos cosméticos. Anvisa., 1.

Bizzo, H. R., Hovell, A. M. C., \& Rezende, C. M. (2009). Óleos essenciais no Brasil: aspectos gerais, desenvolvimento e perspectivas. Química Nova, 32(3), 588-594

Boix, Y. F., Victório, C. P., Lage, C. L. S., \& Kuster, R. M. (2010). Volatile compounds from Rosmarinus officinalis L. and Baccharis dracunculifolia DC. Growing in southeast coast of Brazil. Química Nova, 33(2), 255-257.

Carvalho Junior, R. N. D. (2004). Obtenção de extrato de alecrim (Rosmarinus officinalis) por extração supercrítica: determinação do rendimento global, de parâmetros cinéticos e de equilíbrio e outras variáveis do processo.

Cunha, A. R., Silva, R. S., \& Chorilli, M. (2009). Desenvolvimento e avaliação da estabilidade física de formulações de xampu anticaspa acrescidas ou não de extratos aquosos de hipérico, funcho e gengibre. Revista Brasileira de Farmácia, 90(3), 190-195

da Farmacopéia, Atheneu (2000). Farmacopéia brasileira. In Farmacopéia brasileira.

da Farmacopéia, C. P. D. R. (1988). Farmacopéia brasileira. In Farmacopéia brasileira.

de Castro, K. P. T., da Silva, G. F., \& Madureira, M. T. (2019). Formulação e elaboração de um produto xampu-condicionador de base orgânica na forma sólida/Formulation and elaboration of an organic based xampuproduct in solid form. Brazilian Journal of Development, 5(12), 29575-29587.

Ferreira, A.O. (2008). Guia prático da farmácia magistral. $3^{a}$. ed., São Paulo: Pharmabooks, v.1 409p

Gachkar, L., Yadegari, D., Rezaei, M. B., Taghizadeh, M., Astaneh, S. A., \& Rasooli, I. (2007). Chemical and biological characteristics of Cuminum cyminum and Rosmarinus officinalis essential oils. Food chemistry, 102(3), 898-904.

Isaac, V. L. B., Cefali, L. C., Chiari, B. G., Oliveira, C. C. L. G., Salgado, H. R. N., \& Correa, M. A. (2008). Protocolo para ensaios físico-químicos de estabilidade de fitocosméticos. Journal of Basic and Applied Pharmaceutical Sciences, 29(1).

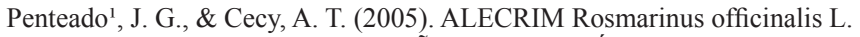
Labiatae (Lamiaceae): UMA REVISÃO BIBLIOGRÁFICA.

Prins, C. L., Lemos, C. S. L., \& Freitas, S. P. (2006). Efeito do tempo de extração sobre a composição e o rendimento do óleo essencial de alecrim (Rosmarinus officinalis). Revista Brasileira de Plantas Medicianais, 8(4), 92-95.

Ribeiro, D. S., Melo, D. B., Guimarães, A. G., \& Velozo, E. S. (2012). Avaliação do óleo essencial de alecrim (Rosmarinus officinalis L.) como modulador da resistência bacteriana. Semina: Ciências Agrárias, 33(2), 687695.

Ruivo, J. S. P. (2012). Fitocosmética: aplicação de extratos vegetais em cosmética e dermatologia (Doctoral dissertation, [sn])

Scacheti, L. F., Matos, N. C., Mallafati, L., \& Navarro, F. F. (2011). Controle de qualidade e análise sensorial em voluntários de xampu esfoliante com extrato hidroalcoólico de Capsicum frutescens L.(Solanaceae). Journal of Basic and Applied Pharmaceutical Sciences, 32(3).

Zanon, A. B. (2010). Aspectos teóricos e práticos sobre a avaliação da estabilidade de emulsões manipuladas em farmácia. 